\title{
EDEMA MACULAR DIABÉTICO CLÍNICAMENTE SIGNIFICATIVO: FACTORES SISTÉMICOS DE RIESGO
}

\section{CLINICALLY SIGNIFICANT DIABETIC MACULAR EDEMA: SYSTEMIC RISK FACTORS}

\author{
ASENSIO-SÁNCHEZ VM${ }^{1}$, GÓMEZ-RAMÍREZ V², MORALES-GÓMEZ I², RODRÍGUEZ-VACA I ${ }^{1}$
}

\section{RESUMEN}

Objetivo: Estudiar los factores de riesgo sistémico del edema macular clínicamente significativo (EMCS) en una población de diabéticos.

Método: Se revisaron las historias clínicas de los pacientes diabéticos diagnosticados de edema macular clínicamente significativo entre 1995 y 2005. Se evaluó la asociación entre EMCS y HgbA1c, comienzo y duración de la diabetes, hipertensión arterial, índice masa corporal, nivel de lípidos, sexo, fumar y excreción urinaria de albúmina. Resultados: 208 ojos fueron considerados con los criterios del estudio. Los pacientes tenían un rango de edad entre 15 y 82 años (media, 66 años) y tenían entre 8 y 64 años (media, 47,5 años) de historia de diabetes. Factores de riesgo significativos para desarrollar EMCS fueron edad avanzada, cifras elevadas de HgbA1c, hipertensión arterial, fumar, valores elevados de colesterol y LDL-colesterol y niveles elevados de proteinuria y microalbuminuria. Conclusiones: Independientemente del tipo de diabetes, los pacientes con diabetes mellitus de larga evolución tienen un elevado riesgo de desarrollar
Objective: We examined the influence of non-ophthalmic parameters as risk factors of clinically significant macular edema (CSME).

Methods: The authors reviewed clinical records of all clinically significant macular edema between 1995 and 2005. The association between the presence of CSME and HgbA1c, onset and duration of diabetes, blood pressure, body mass index, lipid status, sex, tobacco smoking and urinary albumin excretion was evaluated.

Results: 208 eyes met the study criteria. Patients ranged in age from 14 to 82 years (mean, 66 years) and had 8 to 64 years (mean, 47.5 years) of history of diabetes. Significant risk factors for CSME were older age, high levels of HgbA1c, high values of blood pressure, tobacco smoking, high cholesterol and LDL-cholesterol and high levels of proteinuria and microalbuminuria.

Conclusion: Independent on the type of diabetes, patients with long standing diabetes have a high risk to develop diabetic maculopathy, but other closelyrelated risk factors are hypertension, hyperglyce-

\footnotetext{
Recibido: 26/4/07. Aceptado: 18/2/08.

Hospital General. Medina del Campo. Valladolid. España.

1 Doctor en Medicina.

2 Licenciado en Medicina.

Correspondencia:

V.M. Asensio Sánchez

Hospital General Servicio Castellano-Leonés de Salud

Servicio de Oftalmología

Medina del Campo (Valladolid)

España

E-mail: vasensio@hmdc.sacyl.es
} 
maculopatía diabética, pero otros factores de riesgo asociados son la hipertensión, la hiperglucemia, los lípidos, el tabaquismo y el estado renal.

Palabras clave: Diabetes mellitus, maculopatía diabética, EMCS, hiperglucemia, hipertensión, albuminuria, dislipemia, hábito tabáquico. mia, lipids, tobacco smoking and renal status (Arch Soc Esp Oftalmol 2008; 83: 173-176).

Key words: Diabetes mellitus, diabetic maculopathy, CSME, hyperglycemia, hypertension, albuminuria, dyslipemia, tobacco smoke.

\section{INTRODUCCIÓN}

La retinopatía diabética es la primera causa de ceguera legal en los adultos jóvenes de los países occidentales. Aproximadamente 20 millones de personas están afectadas en todo el mundo por esta enfermedad (1,2). En España la prevalencia de DM 2 es entre $4,8 \%$ y $18,7 \%$ y de DM 1 de $0,08 \%$ a $0,2 \%$. La diabetes mellitus y sus patologías asociadas son un problema sanitario de primer orden, y más actualmente con el aumento de la esperanza de vida y de la diabetes infantil en las sociedades desarrolladas $(3,4)$. El edema macular diabético es la causa más frecuente de pérdida grave de visión en el diabético. El edema macular es secundario a la acumulación de fluido en el polo posterior de la retina. Su etiopatogenia no es totalmente comprendida y actualmente no hay una terapia satisfactoria, de aquí la importancia de conocer sus factores de riesgo $(5,6)$. El objetivo de este trabajo es describir, en una población diabética diagnosticada de edema macular clínicamente significativo (EMCS), los factores de riesgo sistémicos asociados.

\section{SUJETOS, MATERIAL Y MÉTODOS}

Este estudio retrospectivo fue aprobado por la Comisión de Investigación y Docencia de nuestro hospital. Se revisaron las historias clínicas de 201 pacientes diabéticos con edema macular clínicamente significativo entre los años 1995 y 2005. En la tabla I se muestran las características demográfi-

Tabla I. Características demográficas

\begin{tabular}{ll}
\hline N. & 201 \\
Sexo (H/M) & $81 / 120$ \\
Edad & 59.2 D.E. 11.3 \\
Edad de inicio de la diabetes & 39 D.E. 10 años \\
Tiempo de evolución de la diabetes & 19 D.E. 10 años \\
\hline
\end{tabular}

H: Hombres; M: Mujeres. cas de la población estudiada. Los criterios de inclusión para los pacientes fueron los siguientes:

- Diabetes mellitus tipo 1 o tipo 2 con EMCS.

- Poder acceder a su historial clínico.

- No haber sido tratado en los tres meses previos al estudio oftalmológico con corticoides, fármacos nefrotóxicos y/o con un proceso quirúrgico.

El diagnóstico de EMCS se basó en el examen mediante lámpara de hendidura con lente de contacto o no contacto. Se estudió el sexo, la edad, tensión arterial media (sistólica y diastólica), obesidad, los lípidos y fumar. El estudio se completó con la determinación de la función renal (microalbuminuria, proteinuria).

Se determinó la hemoglobina glicosilada (Hgb A1c) con un rango establecido por el laboratorio entre $4,7 \%$ y $6,4 \%$.

\section{Definiciones}

- El EMCS se definió según el estudio ETDRS (7) como cualquiera de las siguientes situaciones clínicas:

Grupo I: Exudados duros, asociados a un engrosamiento retiniano adyacente, en el centro de la mácula o a 500 micras.

Grupo II: Engrosamiento retiniano a 500 micras o menos del centro de la mácula.

Grupo III: Engrosamiento retiniano de tamaño igual o mayor a un diámetro papilar con alguna parte de la misma dentro de una región con un diámetro papilar con centro en la fóvea.

- Obesidad: Situación clínica en la que el índice de masa corporal es superior a 30.

- Diabetes tipo 1: diabetes que se desarrolla en pacientes de 30 o menos años.

- Diabetes tipo 2: diabetes que se desarrolla en pacientes de más de 30 años.

- Hipertensión sistólica media: La media de los últimos tres valores de tensión sistólica. 
- Hipertensión diastólica media: La media de los últimos tres valores de tensión diastólica.

- Tabaco:

- No fumador: nunca ha fumado.

- Exfumador: ha dejado el hábito de forma consolidada 1 mes antes del diagnóstico de EMCS.

- Fumador.

- Estudio renal:

- Microalbuminuria: concentración de albúmina en orina en el rango entre $30 \mathrm{mg} / 24 \mathrm{~h}$ y $300 \mathrm{mg} / 24$ $\mathrm{h}$, determinado por radioinmunoensayo.

- Proteinuria: concentración de proteínas en orina $\geq 0,30 \mathrm{~g} / \mathrm{l}$.

En el estudio estadístico se utilizó el test de Mantel-Haenszel para estudiar la significancia de las asociaciones. La t de Student se realizó para comparar las variables contínuas. Con los modelos de regresión se ajustó y estableció el riesgo.

\section{RESULTADOS}

No se consideraron 22 pacientes por estar su historial clínico incompleto. Se estudiaron 208 ojos con EMCS pertenecientes a 179 pacientes diabéticos con un rango de edad comprendido entre $15 \mathrm{y}$ 85 años (media, 66 años). La edad de diagnósticos de su diabetes fue entre 8 y 70 años (media, 49,5 años). El $60 \%$ eran mujeres frente al $40 \%$ que eran varones. El tiempo de duración de la diabetes fue desde 7 a 46 años (media, 38,7 años). La Hgb A1c media era de $9,2 \%$ (rango entre $6,4 \%$ y $19 \%$ ). El $30 \%$ de los casos no tenían retinopatía frente al $70 \%$ que tenía algún grado. En el 26\% (54 ojos) la agudeza visual era de 1 y en el 39,4\% (82 ojos) fue de 0.1 ó menos (tabla II). Según la clasificación de EMCS realizada, el 29,8\% (62 ojos) presentaban un EMCS definible dentro de los conceptos del grupo I, el 38\% (80 ojos) se incluye dentro del grupo II y el 21,6\% (45 ojos) en el grupo III (tabla III). La incidencia de EMCS fue mayor en los diabéticos tipo 2 en tratamiento con insulina $(56,3 \%$ de todos los EMCS) y más baja en los diabéticos tipo 2 en tratamiento con antidiabéticos orales (ADO) (15,6\%). Niveles elevados de Hgb A1c se asociaron significativamente con EMCS, con un incremento del riego por cada $1 \%$ de elevación de 2,4 (IC, 1 , 45-2,78). Se asoció significativamente de forma global la severidad de la retinopatía y EMCS con un riesgo de 1,6 (IC, 1,16-1,83). La patología renal determinada como proteinuria o microalbuminuria y la tensión arterial (sistólica y diastólica) se asociaron significativamente a EMCS con un riesgo de 1,55 (IC, 156-1,78). La edad se asoció a un riesgo de 1,8 (IC, 1,24-2,10). El colesterol total y el colesterol-LDL se asociaron con un riesgo de 1,4 (IC, 1,25-1,67). El fumar (exfumador y fumador) se asoció a un riesgo de 1,50 (IC, 1,26-1,45). El sexo $(0,56, \mathrm{IC}=0,81-1,07)$ y la obesidad $(0,72, \mathrm{IC}=87$ $1,5)$ no fueron factores significativos en el desarrollo de EMCS.

\section{DISCUSIÓN}

La diabetes mellitus es una enfermedad crónica del metabolismo de los hidratos de carbono que daña el riñón, los vasos sanguíneos, nervios periféricos y el aparato visual. La retinopatía diabética causa ceguera en más personas en edad productiva que cualquier otra enfermedad y contribuye grandemente a la ceguera en jóvenes y adultos de los países industrializados $(1,2)$. El aumento de la diabetes en estos países es paralelo al descenso de la salud visual que se observa en los países más avanzados. La rotura de la barrera hemato-retiniana y el resultante aumento de la permeabilidad en la retinopatía diabética es la primera causa de ceguera en estos pacientes $(1,2,4,5)$. En el presente estudio el $26 \%$ de los casos con EMCS tenían agudezas visuales de

Tabla II. Agudeza visual al diagnóstico

\begin{tabular}{lc}
\hline AV & N. ${ }^{\text {ojos }}$ \\
\hline $20 / 20$ & $54(25,9 \%)$ \\
$20 / 30$ & 32 \\
$20 / 40$ & 19 \\
$20 / 80$ & 21 \\
$20 / 200$ & 45 \\
$<20 / 200$ & 30 \\
MM & 4 \\
D & 3 \\
\hline \hline
\end{tabular}

AV: agudeza visual; MM: movimiento de manos; D: dedos.

Tabla III. Clasificación del EMCS

\begin{tabular}{ll}
\hline Grupo EMCS & Ojos $(\mathrm{n} / \%)$ \\
\hline 1 & $62(29,8 \%)$ \\
2 & $80(38,4 \%)$ \\
3 & $45(21,6 \%)$ \\
Mixto & $21(10,09 \%)$ \\
\hline \hline
\end{tabular}

EMCS: edema macular clínicamente significativo. 
1 , frente a un $39 \%$ con agudezas visuales de 0,1 o menos. Es raro el desarrollo de EMCS antes de 10 años de evolución de la diabetes, aunque después se produce un riesgo acumulativo lineal del $6,7 \%$ por año $(8,9)$, otros estudios como el Wisconsin (10) determinaron una incidencia entre los 10-20 años del 3\%. El 56,3\% de nuestros casos de EMCS lo eran en diabéticos tipo 2 en tratamiento con insulina frente al $15,6 \%$ de los diabéticos tipo 2 en tratamiento con antidiabéticos orales, lo cual está de acuerdo con otras series descritas $(7,11)$. En este trabajo se ha pretendido estudiar la relación entre EMCS y factores de riesgo sistémicos. En nuestra muestra existe relación con la edad de diagnóstico de la diabetes, siendo más frecuente el EMCS en las personas de más edad $(7,10)$. En este estudio resulta significativa la relación entre los niveles de HgbA1c y el edema macular, con un incremento del riesgo en más de dos veces por cada $1 \%$ de elevación de hemoglobina glicosilada $(7,9)$. La hipertensión arterial en un diabético aumenta en casi dos veces el riesgo de EMCS. En nuestros pacientes se confirma la relación entre la nefropatía diabética subclínica (microalbuminuria/albuminuria) aumenta en casi dos veces el riesgo de edema macular (12). La dislipemia como alteración metabólica frecuente en el diabético, se estudió con el colesterol y su fracción LDL, su presencia aumenta en más de una vez el riesgo de EMCS $(13,14)$. En este estudio se establece relación entre el hábito tabáquico (presente y pasado) y el EMCS, aumentando en más de una vez el riesgo. En dos variables estudiadas el sexo y la obesidad no se encontraron asociación con EMCS, aunque otras series (8) consideran el sexo masculino como un factor de riesgo añadido para el desarrollo de EMCS. En un estudio reciente Knudsen LL et al (15), en diabéticos tipo 1, no pudieron establecer asociación entre EMCS y los parámetros sistémicos estudiados, pero en los diabéticos tipo 2 encontraron asociación significativa con la duración de la diabetes, HgbA1c, neuropatía y albuminuria. En síntesis, es probable que la incidencia estimada de EMCS esté infravalorada por el alto porcentaje de casos que se presentan en la clínica con una agudeza visual normal, y que ante la sobrecarga de los servicios de oftalmología no se pueda hacer una correcta evaluación microscópica. No debemos olvidar que el mayor porcentaje se da en diabéticos tipo 2 en tratamiento con insulina y que los niveles de Hgb A1c son un factor de riesgo de primer orden por lo que el control metabólico, incluso en fases avanzadas de la diabetes, puede modificar el riesgo de desarrollar EMCS.

\section{BIBLIOGRAFÍA}

1. Klein R, Klein BE, Moss SE. Visual impairment in diabetes. Ophthalmology 1984; 91: 1-9.

2. Antonetti DA, Lieth E, Barber AJ, Gardner TW. Molecular mechanisms of vascular permeability in diabetic retinopathy. Semin Ophthalmol 1999; 14: 240-248.

3. Ruiz-Ramos M, Escolar-Pujolar A, Mayoral-Sanchez E, Corral-San Laureano F, Fernandez-Fernandez I. Mellitus diabetes in Spain: death rates, prevalence, impact, costs and inequalities. Gac Sanit 2006; 20 Suppl 1: 15-24.

4. Lopez-Capape M, Alonso M, Colino E, Mustieles C, Corbaton J, Barrio R. Frequency of the metabolic syndrome in obese Spanish pediatric population. Eur J Endocrinol 2006; 155: 313-319.

5. Joussen AM, Smyth N, Niessen C. Pathophysiology of diabetic macular edema. Dev Ophthalmol 2007; 39: 1-12.

6. Krohne TU, Fauser S, Kirchhof B, Joussen AM. Pathogenesis of diabetic macular edema. Klin Monatsbl Augenheilkd 2003; 220: 521-525.

7. Early Treatment Retinopathy Study Reserach Group. Photocoagulation for diabetic macular edema. Early Diabetic Retinopathy Study report number 1. Arch Ophthalmol 1985; 103: 1796-1806.

8. Kinyoun J, Barton F, Fisher M, Hubbard L, Aiello L, Ferris F 3rd. Detection of diabetic macular edema. Ophthalmoscopy versus photography-Early. Treatment Diabetic Retinopathy Study Report number 5. Ophthalmology 1989; 96: 746-751.

9. Vitale S, Maguire MG, Murphy RP, Hiner CJ, Rourke L, Sackett C, Patz. A. Clinically significant macular edema in type I diabetes. Ophthalmology 1995; 102: 1170-1176.

10. Klein R, Moss SE, Klein BE, Davis MD, DeMets DL. The Wisconsin Epidemiologic Study of Diabetic Retinopathy. XI. The incidence of macular edema. Ophthalmology 1989; 96: 1501-1510.

11. Jeddi Blouza A, Khayati L, Malouche N, Slim B, Azeiz S, Issaoui A, et al. Risk factors for diabetic macular edema. J Fr Ophtalmol 2005; 28: 1033-1038.

12. Girach A, Lund-Andersen H. Diabetic macular oedema: a clinical overview. Int J Clin Pract 2007; 61: 88-97.

13. Rema M, Srivastava BK, Anitha B, Deepa $R$, Mohan V. Association of serum lipids with diabetic retinopathy in urban South Indians-the Chennai Urban Rural Epidemiology Study (CURES) Eye Study-2. Diabet Med 2006; 23: 1029-1036.

14. Miljanovic b, Glynn RJ, Nathan DM, manson JE, Schaumberg DA. A prospective study of serum lipids and risk of diabetic macular edema in type 1 diabetes. Diabetes 2004; 53: 2883-2892.

15. Knudsen LL, Lervang HH, Lundbye-Christensen S, GorstRasmussen A. The North Jutland County Diabetic Retinopathy Study (NCDRS). 2 Non-ophthalmic parameters and clinically significant macular edema. Br J Ophthalmol 2007; 91: 1593-1595. 